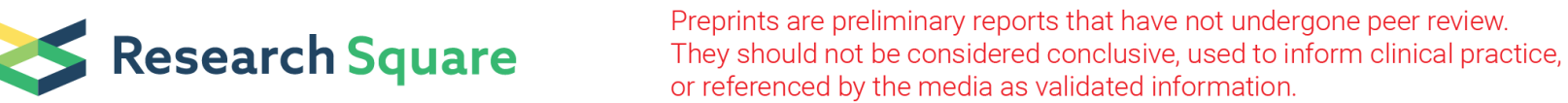

\section{Repetitive transcranial magnetic stimulation promotes neural functional recovery via upregulation of synaptic plasticity-related proteins in rats with traumatic brain injury}

\section{Fangfang Qian}

Southern Medical University https://orcid.org/0000-0001-6628-9183

Youhua He ( $\sim$ 492773924@qq.com)

Southern Medical University

Xiaohui Du

Southern Medical University

Huaxiang Lu

Southern Medical University

Renhong He

Southern Medical University

Jianzhong Fan

Southern Medical University

\section{Research}

Keywords: traumatic brain injury, transcranial magnetic stimulation, brain-derived neurotrophic factor, $\mathrm{N}$ methyl-D-aspartic acid receptor, synaptophysin, synaptic plasticity

Posted Date: August 5th, 2021

DOl: https://doi.org/10.21203/rs.3.rs-757647/v1

License: (c) (i) This work is licensed under a Creative Commons Attribution 4.0 International License. Read Full License 


\section{Abstract \\ Background}

Repetitive transcranial magnetic stimulation (rTMS) has become a popular approach for the treatment of traumatic brain injury (TBI). This study aimed to assess the efficacy and underlying mechanism of rTMS in TBI model rats.

\section{Methods}

Forty-five rats were randomized into SHAM, TBI, and rTMS (TBI and rTMS therapy) groups. Moderate TBI was established using Feeney's weight-dropping method. High-frequency rTMS $(20 \mathrm{~Hz})$ was administered to the damaged area in the rTMS group for two weeks. Neural function was assessed by modified neurological severity score (MNSS) at 3, 9, and 16 days after TBI. Synaptic ultrastructure was observed by transmission electron microscopy and levels of synaptic plasticity-related proteins (BDNF, TrkB, NMDAR1, P-CREB, and SYN) were assessed by immunohistochemistry, Western blotting, and real-time PCR.

\section{Results}

The rTMS group showed a lower MNSS than the TBI group at 16 days $(P<0.05)$. Compared to the TBI group, the postsynaptic density (PSD) was increased, the width of the synaptic cleft was decreased, and the synaptic active zone was lengthened in the rTMS group (all $P<0.05$ ). Compared with the sham group, protein levels and mRNA expression of BDNF, TrkB, NMDAR1, and P-CREB were increased in the TBI group $(P<0.05)$ and further upregulated after rTMS treatment $(P<0.05)$. In addition, rTMS partially reversed downregulation of SYN $(P<0.05)$.

\section{Conclusions}

Taken together, these findings support that rTMS improves neural functional recovery in TBI rats. The possible mechanism is that rTMS modulates synaptic structural plasticity by reducing loss of SYN and alters synaptic functional plasticity by increasing cortical levels of BDNF, TrkB, NMDAR1, and P-CREB.

\section{Background}

Traumatic brain injury (TBI) refers to neuropathologic damage and dysfunction caused by an external force transmitted to the head or body [1]. An estimated 69 million individuals worldwide suffer from TBI each year, making TBI one of the leading causes of death and chronic disability among young people [2]. TBI presents in various forms ranging from mild alterations of consciousness to a continued comatose state and death. TBI patients typically show motor dysfunction, cognitive impairment, and behavioral 
abnormalities after regaining consciousness. In patients with mild injury, neurological dysfunction usually returns to normal within one year; however, in $10-15 \%$ of mild injuries, $50 \%$ of moderate injuries and a greater proportion of severe injuries these defects persist long-term [3]. Thus, TBI poses a heavy burden on the patient's family and society.

For these reasons, it is particularly important to promote early recovery in TBI patients. At present, rehabilitation therapy is the best therapy for neurological deficits after $\mathrm{TBI}$, such as motor rehabilitative training, hyperbaric oxygen therapy, and electro-acupuncture. However, as most TBI survivors still exist neurological impairment after rehabilitation, there is an urgent need for new rehabilitation methods. One new method that has attracted increasing attention is repetitive transcranial magnetic stimulation (rTMS).

rTMS is an efficient and painless brain stimulation technology that generates a super-threshold current in the brain by electromagnetic induction [4]. This method was originally used to explore cortical function in healthy participants [5], but has been subsequently used to treat various neurological and psychiatric disorders including depression [6], bipolar disorder [7], schizophrenia [8], Parkinson's disease [9], and stroke [10]. Recent research has shown that rTMS has the potential to treat TBI [11-13].

TBI has been shown to lead to neuronal cell necrosis, extensive axonal lesions, and physiological and biochemical disorders, resulting in anatomical and functional changes in synaptic transmission $[14,15]$. Modulation of synaptic transmission following TBI likely includes axonal sprouting, synaptogenesis, synaptic remodeling, and synaptic strengthening, which are important ways to promote synaptic plasticity. Some researchers have suggested that rTMS may promote functional recovery of damaged nerve tissue in TBI by enhancing synaptic plasticity [16-18]. rTMS may cause long-lasting changes in cortical excitability. A stimulation frequency $\leq 1 \mathrm{~Hz}$ suppresses cortical excitability and produces longterm depression (LTD), while a stimulation frequency $\geq 5 \mathrm{H}$ facilitates cortical excitability and generates long-term potentiation (LTP) $[19,20]$. LTP and LTD are the main mechanisms for regulating synaptic plasticity at the functional level $[21,22]$, which can be assessed by electrophysiological experiments. Synaptic plasticity also involves remodeling of synaptic structure [23,24], which can be measured by transmission electron microscopy (TEM). However, as there are few studies of rTMS for the treatment of $\mathrm{TBI}$, the underlying mechanism of its benefits remains unclear.

One possible mechanism through which high-frequency rTMS may promote neural functional recovery after TBI is an increase in the expression of synaptic plasticity-related proteins. Specifically, the N-methylD-aspartic acid receptor 1 (NMDAR1) and cAMP response element binding protein (CREB) have been shown to play a key role in synaptic plasticity. Moreover, brain-derived neurotrophic factor (BDNF) is known as an important upstream regulator of LTP. The BDNF-TrkB pathway plays an important role in neural development, maturation, neurogenesis, and survival. Synaptophysin (SYN) is stored in presynaptic vesicles after neuronal synthesis and reflects the number, density, and distribution of synapses. These five proteins involved in synaptic plasticity may have a close relationship with nerve repair following TBI. 
Informed by these prior findings, the present study aimed to further determine the therapeutic effect of rTMS in TBI model rats and to investigate if it promotes neural functional recovery by modulating synaptic plasticity-related proteins.

\section{Materials And Methods}

\section{Animals}

Forty-five male Sprague-Dawley (SD) rats (250-300 g, 8-10 weeks old) were used in this study, because TBI is more common in men than women [25]. The rats were housed in a room at $15-25^{\circ} \mathrm{C}$ and given adequate water and food and experienced $12 \mathrm{~h}$ of light and $12 \mathrm{~h}$ of darkness. The model of moderate TBI was established by Feeney's weight-dropping method [26]. After anesthesia, shaving, and disinfection, the scalp was incised at the middle of the sagittal plane to separate the periosteum. We took the posterior 1.5 $\mathrm{mm}$ and dextral $2.5 \mathrm{~mm}$ of the bregma at the right hemisphere as the center, opened a round window with a diameter of $5 \mathrm{~mm}$, and kept the dura mater intact. An impact force of $600 \mathrm{~g} / \mathrm{cm}$ was caused by a $20 \mathrm{~g}$ weight falling from a height of $30 \mathrm{~cm}$, which struck the dura and caused moderate TBI. Next, we closed the bone window with bone wax and sutured the scalp. All of these steps were completed by experienced researchers within two days. After recovery from anesthesia, the animals were put back into their cages and provided with water and food. The scalps of rats in the SHAM group were sutured immediately after skull incision to ensure integrity of the brain parenchyma.

\section{Behavioral Evaluation}

Behavior was assessed using the modified neurological severity score (MNSS) at 3, 9, and 16 days after TBI. MNSS is composed of motor, sensory, balance, and reflex tests. Scores are as follows: mild neurological injury, 1-6; moderate neurological injury, 7-12; and severe neurological dysfunction, 13-18. The higher the score, the more serious the injury. Each rat was scored separately by two experimenters. Rats with scores of 7-12 (moderate injury) at three days after TBI were selected for subsequent experiments.

\section{Experimental Grouping}

Rats were randomly divided into three groups: SHAM group $(n=15)$, TBI group $(n=15)$, and rTMS group (TBI and rTMS therapy, $n=15$ ). Rats in the rTMS group received 800 stimuli at $30 \%$ of the relaxed motor threshold (RMT) per day, resulting in 40 trains at $20 \mathrm{~Hz}$ for $1 \mathrm{~s}$, with $15 \mathrm{~s}$ intervals between trains [27]. Starting from 4 days after TBI, rTMS was performed for 10 days during a 2-week period. The coil was placed above the injured site, close to the scalp. In the SHAM and TBI groups, the rats were immobilized under the coil but not given stimulation.

\section{Transmission Electron Microscopy}

Sixteen days after TBI, rats ( $n=6 /$ group) from the three groups were anesthetized and perfused with normal saline until a colorless fluid flowed from the mouth and nose. The brain tissue specimens of each 
rat were separated from a similar position around the damaged area and stored in $4 \%$ glutaraldehyde. Next, the tissue specimens were trimmed to approximately $1 \mathrm{~mm}^{3}$, fixed using $1 \%$ osmic acid, dehydrated in acetone, and embedded in epoxy resin Epon-812. Sections with a thickness of $80 \mathrm{~nm}$ were prepared using an ultrathin section mechanism. The sections were double-stained with $2 \%$ uranium acetate and lead citrate. Images were observed and recorded using a Hitachi 7700 transmission electron microscope (Electron microscope Experimental Center of Southern Medical University, Guangzhou, China).

\section{Immunohistochemistry}

Sixteen days after TBI, rats ( $n=6 /$ group) were anesthetized and their brains were fixed in $4 \%$ paraformaldehyde for more than $24 \mathrm{~h}$. Specimens were dehydrated in turn with alcohol and then embedded in paraffin wax. Five sections ( $3 \mu \mathrm{m}$ thick) taken from the injury site in each rat were used for immunohistochemistry staining. The sections were incubated overnight at $4^{\circ} \mathrm{C}$ with rabbit BDNF $(1: 2000$, Abcam), TrkB (1:250, Abcam), NMDAR1 (1:200, Abcam), P-CREB (1:100, Abcam), and SYN (1:2000, Abcam). Next, the sections were rinsed in TBST three times, each time lasting $5 \mathrm{~min}$. The sections were incubated with secondary antibody at $37^{\circ} \mathrm{C}$ for $45 \mathrm{~min}$ and $50 \mu \mathrm{L}$ diaminobenzidine (DAB) was added. After redyeing with hematoxylin, the sections were dehydrated and sealed. The level of antibodies in each group was observed under an optical microscope (Olympus \# CX31) at 400x magnification.

\section{Western Blotting}

Sixteen days after TBI, the rats ( $n=6 /$ group) were sacrificed. Tissues in the ipsilateral hemisphere (excluding the damaged area) were selected for Western blotting. The protein was homogenized in RIPA lysis buffer and the protein concentration was measured by the bicinchoninic acid method. Samples were separated by sodium dodecyl sulfate-polyacrylamide gel electrophoresis (SDS-PAGE) and transferred to polyvinylidene fluoride membranes (PVDF). The blots were blocked with $5 \%$ skimmed milk for $1 \mathrm{~h}$ at room temperature, followed by incubation with the following rabbit primary antibodies: anti-BDNF (1:1000, Abcam), TrkB (1:1000, Abcam), NMDAR1 (1:10000, Abcam), P-CREB (1:10000, Abcam), and SYN $(1: 80000, A b c a m)$ at $4^{\circ} \mathrm{C}$ overnight. After washing with TBST, the membranes were incubated with secondary antibody for $1 \mathrm{~h}$ at room temperature. Next, they were put into the photo-chemiluminescence gel imaging system to determine the corresponding protein band strength. The bands were quantitated using Image J analysis software $(\mathrm{NIH})$. GAPDH was used as an internal reference to calculate the relative expression of each antibody.

\section{Real-Time Polymerase Chain Reaction (RT-PCR)}

Sixteen days after TBI, the rats ( $n=6 /$ group) were sacrificed. After removing the damaged area, total RNA was extracted from the affected cerebral hemispheres using TRIzol reagent. An equal amount of RNA was converted into cDNA using RevertAid reverse transcriptase (Thermo Fisher). Using the GAPDH gene as the internal reference, the mRNA expression of the BDNF, TrkB, NMDAR1, P-CREB, and SYN genes was detected by PCR. For each gene, two specific PCR primers were used: $B D N F$ (forward: AGAATGACAAGGCATAGGAAGGT and reverse: CCAAGAGGTAAAGTGTAGAAGGGA), Ntrk2 (forward: GGATTTGGTGTACCGAGCCT and reverse: TTGATGTGCCGCAGGTTG), Grin 1 (forward: 
CGTGGGCAACACCAACATC and reverse: AGTCACTCCGTCCGCATACTT), CREB (forward: GACAACCAGCAGAGTGGAGAT

Gand reverse: TTACAGTGGGAGCAGATGACG), Syp1 (forward: TGGGGTTCAGGGATGATC

TAA and reverse: GAGTACGCCCTCCTGCTTTTA), and GAPDH (forward : CGCTAACATCAA

ATGGGGTG and reverse: TTGCTGACAATCTTGAGGGAG). RT-PCR reactions were performed in a $20 \mu \mathrm{L}$ total volume with $5 \mu \mathrm{L}$ cDNA diluted 10 times, $0.4 \mu \mathrm{L}$ each forward and reverse primers, $10 \mu \mathrm{L}$ AceQ Universal SYBR qPCR Master Mix (2x mix), and $4.2 \mu \mathrm{L} \mathrm{ddH2O}$. The amplification protocol consisted of one cycle at $95^{\circ} \mathrm{C}$ for $5 \mathrm{~min}$ followed by 40 cycles at $95^{\circ} \mathrm{C}$ for $10 \mathrm{~s}$ and $60^{\circ} \mathrm{C}$ for $10 \mathrm{~s}$. GAPDH was used as an internal control. The formula $2-\Delta \Delta C T$ was used to indicate the relative expression level of each gene.

\section{Statistical Analysis}

Data were analyzed using SPSS 25.0 software. All results were reported as mean \pm standard deviation (SD). Under the condition of determining the homogeneity of variance, the independent sample t-test or one-way analysis of variance (ANOVA) was selected. For the latter, pairwise comparisons were performed using the least significant difference (LSD) test. The level of statistical significance was set at $\mathrm{P}<0.05$.

\section{Results}

\section{Model Assessment}

On the third day after TBI, all rats underwent MRI examination (T2M1; Fig. 1). The average area of brain parenchyma damage did not differ significantly between the TBI and rTMS groups. Moreover, there were no differences in body weight (Fig. 2A) or MNSS values (Fig. 2B) between the two groups. These data support that the TBI model is stable.

\section{Effects of rTMS on Neural Function}

To evaluate whether rTMS can improve neurological dysfunction induced by TBI, MNSS was compared at 3,9 , and 16 days after TBI. Compared with the SHAM group, the TBI group showed obvious functional defects that improved after rTMS treatment. Furthermore, the MNSS of the TMS group was significantly lower than that of the TBI group at 16 days (Fig. 3, $\mathrm{P}<0.05$ ).

\section{Effects of rTMS on Synaptic Structural Plasticity}

The thickness of postsynaptic density (PSD), the width of the synaptic cleft, and the length of the synaptic active zone (AZ) were calculated in the three groups. Compared to the TBI group, the PSD thickness was increased, the width of the synaptic cleft was decreased, and the synaptic AZ was lengthened in the rTMS group (Fig. 4, all P<0.05). 
The protein level of SYN was detected at 16 days after TBI by immunochemistry and western blot assay. Compared with the SHAM group, weak immunopositive staining was observed in the TBI group. After rTMS treatment, strong immunohistochemical staining for SYN was observed in the cytoplasm of cells around the injury site. Compared with the SHAM group, the protein level of SYN was significantly reduced in the TBI group, indicating loss of synapses. rTMS treatment resulted in a significantly higher level of SYN than in the TBI group. The mRNA expression of SYN in the TBI group was significantly lower than that in the SHAM group, and was partially upregulated after rTMS treatment (Fig. 5).

Table 1

Comparison of synaptic parameters in the three groups.

\begin{tabular}{|llll|}
\hline & SHAM & TBI & rTMS \\
\hline Number of synapses & 50 & 50 & 50 \\
\hline Thickness of PSD $(\mathrm{nm})$ & $52.39 \pm 6.58^{\star}$ & $44.62 \pm 5.70$ & $50.07 \pm 6.25^{\star}$ \\
\hline Width of the synaptic cleft $(\mathrm{nm})$ & $15.15 \pm 3.02^{\star}$ & $18.65 \pm 3.85$ & $16.20 \pm 3.23^{\star}$ \\
\hline Length of the active zone $(\mathrm{nm})$ & $340.23 \pm 30.31^{\star}$ & $285.27 \pm 22.27$ & $334.19 \pm 28.53^{\star}$ \\
\hline Values are mean $\pm \mathrm{SD} ; * \mathrm{P}<0.05$ versus the TBI group. & \\
\hline
\end{tabular}

\section{Effects of rTMS on Synaptic Functional Plasticity}

The protein levels of BDNF, TrkB, NMDAR1, and P-CREB in the ipsilateral cerebral cortex were assessed at 16 days after TBI by immunochemistry. Compared with the SHAM group, BDNF, TrkB, NMDAR1, and PCREB in the cytoplasm of cells around the injury site in the TBI group showed strong immunohistochemical staining. The number of positive cells increased after rTMS treatment (Fig. 6).

Cortical tissues in the ipsilateral hemisphere excluding the damaged area were used for Western blotting and RT-PCR at 16 days after TBI. The protein levels and mRNA expression of BDNF, TrkB, NMDAR1, and PCREB are shown in Figs. 7 and 8, respectively. The results showed that the protein levels and mRNA expression of BDNF, TrkB, NMDAR1, and P-CREB in the TBI group were higher than those in the SHAM group. Notably, levels were further upregulated after rTMS treatment.

\section{Discussion}

In the present study, our results revealed that the expression of synaptic plasticity related proteins (BDNF, TrkB, NMDAR1, P-CREB, and SYN) in the cerebral cortex was significantly increased by high-frequency rTMS. In combination with these neuroprotective effects, we demonstrated that high-frequency rTMS improved functional recovery in TBI rats, as supported by a reduction in MNSS value, suggesting that this method is a promising strategy for TBI rehabilitation. 
After TBI, synaptic morphological structure is seriously damaged. The reshaping of synaptic morphology is considered an important aspect of synaptic plasticity. In the present study, we found that increased PSD thickness, decreased width of the synaptic cleft, and lengthened synaptic active zone after rTMS treatment in TBI rats. Furthermore, we found that rTMS reduced the loss of SYN. SYN exists widely in nerve endings in the central and peripheral nervous system and is specifically distributed in presynaptic vesicle membranes. It is considered a marker protein of presynaptic terminals and has been extensively used to quantify the number, density, and distribution of synapses $[28,29]$. Therefore, our findings suggest that high-frequency rTMS may modify synaptic structure by increasing SYN levels in TBI rats. If rTMS does enhance the plasticity of synaptic function in TBI rats the mechanism is still unclear, which was the focus of subsequent analyses in this study.

NMDA receptors are thought to play a bidirectional role in the occurrence and development of TBI. In the acute phase, excitotoxicity caused by glutamate accumulation is mainly mediated by NMDA receptors, which leads to secondary neuronal injury and various behavioral dysfunctions. However, in the subacute and chronic phases, NMDA receptors activation can resist synaptic transmission disturbances caused by the continuous increase in inhibitory neurotransmitters. NMDA receptors are an important mediator of brain plasticity and can transform specific neuronal activity patterns into changes in synaptic structure and function [30], which is considered the basis of changes in behavioral function. NMDAR1 is present in all endogenous NMDARs and is widely expressed throughout development [31,32]. Activation of NMDAR1 in the hippocampus CA1 area is known to play an important role in LTP induction related to learning and memory ability [33-35]. In the present study, we found that the NMDAR1 protein level in the cerebral cortex increased after rTMS treatment in TBI rats. Furthermore, the neurological function of TBI rats significantly improved after rTMS treatment. Therefore, we speculate that rTMS enhances NMDAR1 protein expression in the subacute phase of TBI and then promotes NMDARs-dependent LTP, which changes synaptic transmission effectiveness, thereby promoting neural functional recovery in rats.

High-frequency rTMS activates NMDA receptors and leads to massive $\mathrm{Ca} 2+$ influx. $\mathrm{Ca} 2+$ and calmodulin form a $\mathrm{Ca} 2+$ / calmodulin complex that eventually triggers cAMP response element binding protein (CREB) phosphorylation [36, 37]. CREB is a general transcription factor that is involved in various cellular activities. Classically, LTP maintenance is split into early and late phases: the early stage requires kinase activation, whereas the late, stable phase requires protein synthesis. CREB is thought to be needed to initiate the transcription of proteins required for long-lasting plasticity [38]. For example, LTP does not last $>90 \mathrm{~min}$ in the hippocampus of animals lacking most of the major isoforms of CREB [39]. CREB can also drive axonal growth from neurons on inhibitory substrates $[40,41]$ and participates in the regulation of neurogenesis in the subventricular zone [42]. Moreover, high levels of phosphorylated CREB (P-CREB) have been observed in newly generated, immature neurons of the subgranular (SGZ) and subependymal ventricular zone/olfactory bulb (SVZ/OB) system [42]. P-CREB can also block the transcription of inflammatory mediators by regulating P-NF-KB and reduces the activation of proinflammatory microglia $[43,44]$. Overall, our findings indicate that high-frequency rTMS enhanced expression of the CREB gene and the level of p-CREB protein, inducing the occurrence of late LTP (L-LTP) and ultimately playing a neuroprotective role in TBI rats. 
In addition, p-CREB activates BDNF transcription by binding to a key $\mathrm{C} 2$ + response element (CRE) in the $B D N F$ gene $[45,46]$. BDNF belongs to the neurotrophin family, which maintains high expression levels in various brain regions including rodent and human cerebral cortex and hippocampus. Not only does BDNF regulate neuronal development, growth, and survival, it also plays a role in synaptic transmission and LTP $[47,48]$. Furthermore, a large body of evidence suggests that BDNF is essential for L-LTP $[49,50]$. BDNF may activate synaptic consolidation through transcription and rapid dendritic trafficking of mRNA encoded by the immediate early gene, Arc. [51, 52], thus making LTP exist in a more stable state. Significant L-LTP damage has been observed in hippocampal slices of mice treated with BDNF functionblocking monoclonal antibody [53] or BDNF gene knockout [54]. Since P-CREB regulates BDNF gene transcription, consistent with previous results, we observed that rTMS treatment increased BDNF protein levels following brain injury. BDNF also results in autophosphorylation of intracellular tyrosine residues mainly by binding to tropomyosin receptor kinase $B(T r k B)$, which initiates various intracellular signaling pathways including the phosphatidylinositol 3 kinase (PI3K) and mitogen-activated protein kinase/extracellular signal-regulated kinase (MAPK/ERK). These pathways can enhance the phosphorylation of CREB at Ser133 $[55,56]$ and activate NMDAR1 $[57,58]$, resulting in positive feedback to facilitate LTP. They can also promote dendritic growth and branching by regulating protein synthesis and cytoskeleton development [59]. Moreover, the PI3K pathway activates protein kinase B (Akt), which ultimately promotes cell survival by inhibiting the Bcl-2 associated death promotor (Bad) and, consequently, enabling the expression of anti-apoptotic proteins such as BcL2 [60, 61]. Thus, maintenance of LTP and improvement in neural function in TBI rats are closely related to the increase in BDNF protein level and activation of the BDNF-TrkB pathway by rTMS.

This study has several limitations. The first limitation is that we did not apply rTMS to normal rats. Whether the rTMS effect observed in TBI rats can be replicated in normal rats remains to be investigated. Second, although we used the smallest round coils available, the size of the coil was still larger than the rat brain. Thus, we could not ensure that stimulation was applied to a specific brain region. Third, as rats were only observed for two weeks after TBI, the subsequent therapeutic effects of rTMS are unclear. Fourth, in the experiment, we only used MNSS to evaluate the neural function of rats, and MNSS values are greatly influenced by subjective factors. Finally, as our sample size was small, the experimental results should be confirmed in large-scale studies.

\section{Conclusions}

In conclusion, our findings suggest that rTMS may promote the recovery of neurological function in TBI rats through the following mechanisms: (1) rTMS can modulate synaptic structural plasticity: rTMS enhances the SYN protein level in the presynaptic region and promotes synaptic reconstruction. (2) rTMS affects synaptic functional plasticity: rTMS increases the opening frequency of the NMDA receptors channel in the postsynaptic membrane and promotes the occurrence of NMDA receptor-dependent LTP. When the NMDA receptor is activated, the influx of massive amounts of $\mathrm{Ca} 2+$ triggers the phosphorylation of CREB, which ultimately regulates BDNF transcription. The BDNF-TrkB pathway 
reversely activates CREB and NMDAR1, resulting in positive feedback and making LTP more stable and durable.

\section{Abbreviations}

TBI: traumatic brain injury; rTMS: Repetitive transcranial magnetic stimulation; LTD: long-term depression; LTP: long-term potentiation; TEM: transmission electron microscopy; NMDAR1: N-methyl-D-aspartic acid receptor 1; CREB: CAMP response element binding protein; BDNF: brain-derived neurotrophic factor; SYN: Synaptophysin; SD: Sprague-Dawley; MNSS: modified neurological severity score; RMT: relaxed motor threshold; DAB: diaminobenzidine; SDS-PAGE: sodium dodecyl sulfate-polyacrylamide gel electrophoresis; PVDF: polyvinylidene fluoride; ANOVA: one-way analysis of variance; LSD: least significant difference; PSD: postsynaptic density; AZ: active zone; P-CREB: phosphorylated CREB; SGZ: subgranular zone; SVZ/OB: subependymal ventricular zone/olfactory bulb; TrkB: tropomyosin receptor kinase B; PI3K: phosphatidylinositol 3 kinase; MAPK/ERK: mitogen-activated protein kinase/extracellular signal-regulated kinase.

\section{Declarations}

\section{Acknowledgements}

The authors would like to thank all the participants who took part in this study.

\section{Authors' contributions}

Dr. Renhong He and Dr. Jianzhong Fan both contributed to the concept and design of the study. Dr. Fangfang Qian, Dr. Xiaohui Du and Dr. Huaxiang Lu completed the animal experiments and data acquisition. Dr. Fangfang Qian and Dr. Youhua He completed data analysis and drafting and revising of the manuscript. All authors read and approved the final version of this manuscript.

\section{Funding}

This work was supported by the Southern Medical University of Nanfang Hospital Dean fund (Grant No. 50107021).

\section{Availability of data and materials}

Raw data in this study is available from the corresponding author on reasonable request.

\section{Ethics approval}

The experimental work was performed in accordance with the guidelines for the Care and Use of Laboratory Animals from the National Institute of Health (Publication No. 8023, revised 1978) and approved by the Institutional Animal Ethical Committee of Southern Medical University. 


\section{consent to participate}

Not applicable.

\section{Consent for publication}

Not applicable.

\section{Competing interests}

The authors declare no conflict of interest.

\section{Author details}

a Department of Rehabilitation Medicine, Nanfang Hospital, Southern Medical University, Guangzhou 510515, China; ' Department of Comprehensive Ward, Nanfang Hospital, Southern Medical University, Guangzhou 510515, China; * Correspondence to: Renhong He and Jianzhong Fan. Department of Rehabilitation Medicine, Nanfang Hospital, Southern Medical University, 1838 Guangzhou North Avenue, 510515, China.

\section{References}

1. Mckee AC, Daneshvar DH. The neuropathology of traumatic brain injury. Handb Clin Neurol. $2015 ; 127: 45-66$.

2. Dewan $\mathrm{MC}$, et al., Estimating the global incidence of traumatic brain injury. Journal of neurosurgery, 2018: p. 1-18.

3. Hoskison MM, et al. Persistent working memory dysfunction following traumatic brain injury: evidence for a time-dependent mechanism. Neuroscience. 2009;159(2):483-91.

4. Seewoo BJ, et al. Combined rTMS/fMRI Studies: An Overlooked Resource in Animal Models. Front NeuroSci. 2018;12:180.

5. Latorre A, et al. The use of transcranial magnetic stimulation as a treatment for movement disorders: A critical review. Movement disorders: official journal of the Movement Disorder Society. 2019;34(6):769-82.

6. Perera T, et al. The Clinical TMS Society Consensus Review and Treatment Recommendations for TMS Therapy for Major Depressive Disorder. Brain Stimul. 2016;9(3):336-46.

7. Oldani L, et al. Brain stimulation treatments in bipolar disorder: A review of the current literature. The world journal of biological psychiatry: the official journal of the World Federation of Societies of Biological Psychiatry. 2016;17(7):482-94.

8. Dougall N, et al. Transcranial Magnetic Stimulation for Schizophrenia. Schizophr Bull. 2015;41(6):1220-2. 
9. Chen KS, Chen R. Invasive and Noninvasive Brain Stimulation in Parkinson's Disease: Clinical Effects and Future Perspectives. Clin Pharmacol Ther. 2019;106(4):763-75.

10. Fisicaro F, et al. Repetitive transcranial magnetic stimulation in stroke rehabilitation: review of the current evidence and pitfalls. Ther Adv Neurol Disord. 2019;12:1756286419878317.

11. Dhaliwal SK, Meek BP, Modirrousta MM. Non-Invasive Brain Stimulation for the Treatment of Symptoms Following Traumatic Brain Injury. Frontiers in psychiatry. 2015;6:119.

12. Pape TL, Rosenow J, Lewis G. Transcranial magnetic stimulation: a possible treatment for TBI. J Head Trauma Rehabil. 2006;21(5):437-51.

13. Nardone R, et al. Repetitive transcranial magnetic stimulation in traumatic brain injury: Evidence from animal and human studies. Brain research bulletin. 2020;159:44-52.

14. Ng SY, Lee A. Traumatic Brain Injuries: Pathophysiology and Potential Therapeutic Targets. Frontiers in cellular neuroscience. 2019;13:528.

15. Algattas $\mathrm{H}$, Huang JH. Traumatic Brain Injury pathophysiology and treatments: early, intermediate, and late phases post-injury. Int J Mol Sci. 2013;15(1):309-41.

16. Nardone R, et al. Repetitive transcranial magnetic stimulation in traumatic brain injury: Evidence from animal and human studies. Brain research bulletin. 2020;159:44-52.

17. Villamar MF, et al. Noninvasive brain stimulation to modulate neuroplasticity in traumatic brain injury. Neuromodulation: journal of the International Neuromodulation Society. 2012;15(4):326-38.

18. Bolognini N, Pascual-Leone A, Fregni F. Using non-invasive brain stimulation to augment motor training-induced plasticity. J Neuroeng Rehabil. 2009;6:8.

19. Page SJ, et al. It takes two: noninvasive brain stimulation combined with neurorehabilitation. Arch Phys Med Rehabil. 2015;96:S89-93.

20. Iglesias AH. Transcranial Magnetic Stimulation as Treatment in Multiple Neurologic Conditions. Curr Neurol Neurosci Rep. 2020;20(1):1.

21. Levy M, et al. Neurotrophic factors and neuroplasticity pathways in the pathophysiology and treatment of depression. Psychopharmacology. 2018;235(8):2195-220.

22. Koller EJ, Chakrabarty P. Tau-Mediated Dysregulation of Neuroplasticity and Glial Plasticity. Front Mol Neurosci. 2020;13:151.

23. Michelini LC, Stern JE. Exercise-induced neuronal plasticity in central autonomic networks: role in cardiovascular control. Exp Physiol. 2009;94(9):947-60.

24. Wobrock T, Hasan A, Falkai P. Innovative treatment approaches in schizophrenia enhancing neuroplasticity: aerobic exercise, erythropoetin and repetitive transcranial magnetic stimulation. Curr Pharm Biotechnol. 2012;13(8):1595-605.

25. Gupte R, et al. Sex Differences in Traumatic Brain Injury: What We Know and What We Should Know. J Neurotrauma. 2019;36(22):3063-91.

26. Feeney DM, et al. Responses to cortical injury: I. Methodology and local effects of contusions in the rat. Brain research. 1981;211(1):67-77. 
27. Luo J, et al. High-Frequency Repetitive Transcranial Magnetic Stimulation (rTMS) Improves Functional Recovery by Enhancing Neurogenesis and Activating BDNF/TrkB Signaling in Ischemic Rats. Int J Mol Sci. 2017;18(2):455.

28. Ji S, et al., The Role of Acupuncture Improving Cognitive Deficits due to Alzheimer's Disease or Vascular Diseases through Regulating Neuroplasticity. Neural plasticity, 2021. 2021: p. 8868447.

29. Lin J, et al., Effect of Tetramethylpyrazine on Neuroplasticity after Transient Focal Cerebral Ischemia Reperfusion in Rats. Evidence-based complementary and alternative medicine: eCAM, 2021. 2021: p. 1587241.

30. Piubelli L, et al. The Role of D-Amino Acids in Alzheimer's Disease. Journal of Alzheimer's disease: JAD. 2021;80(2):475-92.

31. Zhang C, et al. Prenatal Deltamethrin Exposure-Induced Cognitive Impairment in Offspring Is Ameliorated by Memantine Through NMDAR/BDNF Signaling in Hippocampus. Front NeuroSci. 2018;12:615.

32. Ju P, Cui D. The involvement of N-methyl-D-aspartate receptor (NMDAR) subunit NR1 in the pathophysiology of schizophrenia. Acta Biochim Biophys Sin. 2016;48(3):209-19.

33. Harris EW, Ganong AH, Cotman CW. Long-term potentiation in the hippocampus involves activation of N-methyl-D-aspartate receptors. Brain research. 1984;323(1):132-7.

34. Morris RG, et al. Selective impairment of learning and blockade of long-term potentiation by an Nmethyl-D-aspartate receptor antagonist, AP5. Nature. 1986;319(6056):774-6.

35. Kumar A, Thinschmidt JS, Foster TC. Subunit contribution to NMDA receptor hypofunction and redox sensitivity of hippocampal synaptic transmission during aging. Aging. 2019;11(14):5140-57.

36. Wang H, Peng RY. Basic roles of key molecules connected with NMDAR signaling pathway on regulating learning and memory and synaptic plasticity. Military Medical Research. 2016;3(1):26.

37. Gandolfi D, et al., Activation of the CREB/c-Fos Pathway during Long-Term Synaptic Plasticity in the Cerebellum Granular Layer. Frontiers in Cellular Neuroscience, 2017. 11.

38. Glazewski S, et al., Impaired experience-dependent plasticity in barrel cortex of mice lacking the alpha and delta isoforms of CREB. Cerebral cortex (New York, N.Y.: 1991), 1999. 9(3): p. $249-56$.

39. Barth AL, et al. Upregulation of CAMP response element-mediated gene expression during experiencedependent plasticity in adult neocortex. The Journal of neuroscience: the official journal of the Society for Neuroscience. 2000;20(11):4206-16.

40. Ma TC, et al. cAMP-responsive element-binding protein (CREB) and cAMP co-regulate activator protein 1 (AP1)-dependent regeneration-associated gene expression and neurite growth. J Biol Chem. 2014;289(47):32914-25.

41. Caracciolo L, et al. CREB controls cortical circuit plasticity and functional recovery after stroke. Nature communications. 2018;9(1):2250.

42. Lu KT, et al. NKCC1 mediates traumatic brain injury-induced hippocampal neurogenesis through CREB phosphorylation and HIF-1a expression. Pflug Arch: Eur J Physiol. 2015;467(8):1651-61. 
43. Rehman SU, et al., Neurological Enhancement Effects of Melatonin against Brain Injury-Induced Oxidative Stress, Neuroinflammation, and Neurodegeneration via AMPK/CREB Signaling. Cells, 2019. $8(7)$.

44. Bhat SA, et al. Enhanced Akt/GSK-3ß/CREB signaling mediates the anti-inflammatory actions of mGluR5 positive allosteric modulators in microglia and following traumatic brain injury in male mice. Journal of neurochemistry. 2021;156(2):225-48.

45. Yan X, et al. CaMKII-Mediated CREB Phosphorylation Is Involved in Ca2+-Induced BDNF mRNA Transcription and Neurite Outgrowth Promoted by Electrical Stimulation. PloS one. 2016;11(9):e0162784.

46. Esvald EE, et al. CREB Family Transcription Factors Are Major Mediators of BDNF Transcriptional Autoregulation in Cortical Neurons. The Journal of neuroscience: the official journal of the Society for Neuroscience. 2020;40(7):1405-26.

47. Miranda M, et al. Brain-Derived Neurotrophic Factor: A Key Molecule for Memory in the Healthy and the Pathological Brain. Frontiers in cellular neuroscience. 2019;13:363.

48. Numakawa T, Odaka H, Adachi N. Actions of Brain-Derived Neurotrophin Factor in the Neurogenesis and Neuronal Function, and Its Involvement in the Pathophysiology of Brain Diseases. International journal of molecular sciences, 2018. 19(11).

49. Panja D, Bramham CR. BDNF mechanisms in late LTP formation: A synthesis and breakdown. Neuropharmacology, 2014: p. 664-76.

50. Santi S, et al. Hippocampal neurons recycle BDNF for activity-dependent secretion and LTP maintenance. EMBO J. 2006;25(18):4372-80.

51. Bramham CR, Messaoudi E. BDNF function in adult synaptic plasticity: the synaptic consolidation hypothesis. Progress in neurobiology. 2005;76(2):99-125.

52. Wibrand $\mathrm{K}$, et al. Identification of genes co-upregulated with Arc during BDNF-induced long-term potentiation in adult rat dentate gyrus in vivo. Eur J Neurosci. 2006;23(6):1501-11.

53. Chen $\mathrm{G}$, et al. Relative contribution of endogenous neurotrophins in hippocampal long-term potentiation. The Journal of neuroscience: the official journal of the Society for Neuroscience. 1999;19(18):7983-90.

54. Patterson SL, et al. Recombinant BDNF rescues deficits in basal synaptic transmission and hippocampal LTP in BDNF knockout mice. Neuron. 1996;16(6):1137-45.

55. Li N, Liu GT. The novel squamosamide derivative FLZ enhances BDNF/TrkB/CREB signaling and inhibits neuronal apoptosis in APP/PS1 mice. Acta pharmacologica Sinica. 2010;31(3):265-72.

56. Liu W, et al., Brain-Derived Neurotrophic Factor and Its Potential Therapeutic Role in Stroke Comorbidities. Neural plasticity, 2020. 2020: p. 1969482.

57. Zhang HY, Jin XB, Lue TF. Three important components in the regeneration of the cavernous nerve: brain-derived neurotrophic factor, vascular endothelial growth factor and the JAK/STAT signaling pathway. Asian journal of andrology. 2011;13(2):231-5. 
58. Lin D, et al. The neuroprotective role of acupuncture and activation of the BDNF signaling pathway. Int J Mol Sci. 2014;15(2):3234-52.

59. Kowiański P, et al. BDNF: A Key Factor with Multipotent Impact on Brain Signaling and Synaptic Plasticity. Cell Mol Neurobiol. 2018;38(3):579-93.

60. Wurzelmann M, Romeika J, Sun D. Therapeutic potential of brain-derived neurotrophic factor (BDNF) and a small molecular mimics of BDNF for traumatic brain injury. Neural Regen Res. 2017;12(1):712.

61. Keefe KM, Sheikh IS, Smith GM. Targeting Neurotrophins to Specific Populations of Neurons: NGF, BDNF, and NT-3 and Their Relevance for Treatment of Spinal Cord Injury. International journal of molecular sciences, 2017. 18(3).

\section{Figures}

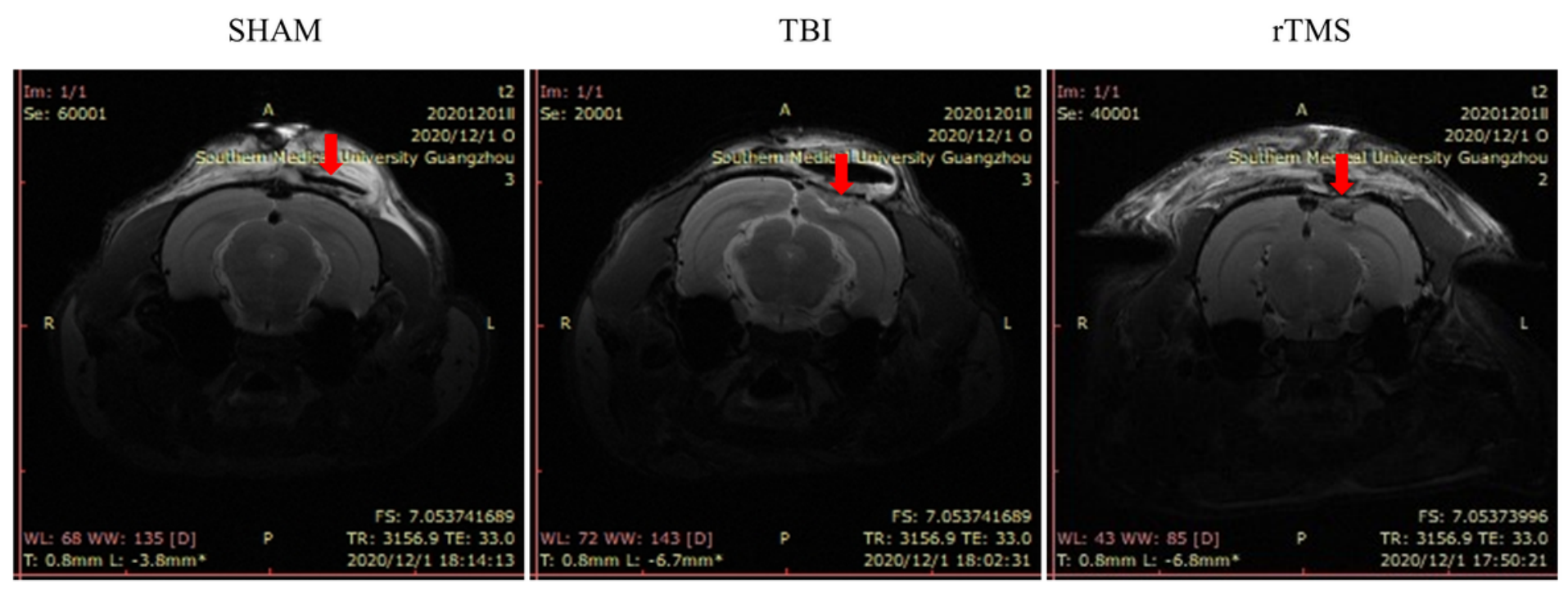

\section{Figure 1}

MRI examination. 

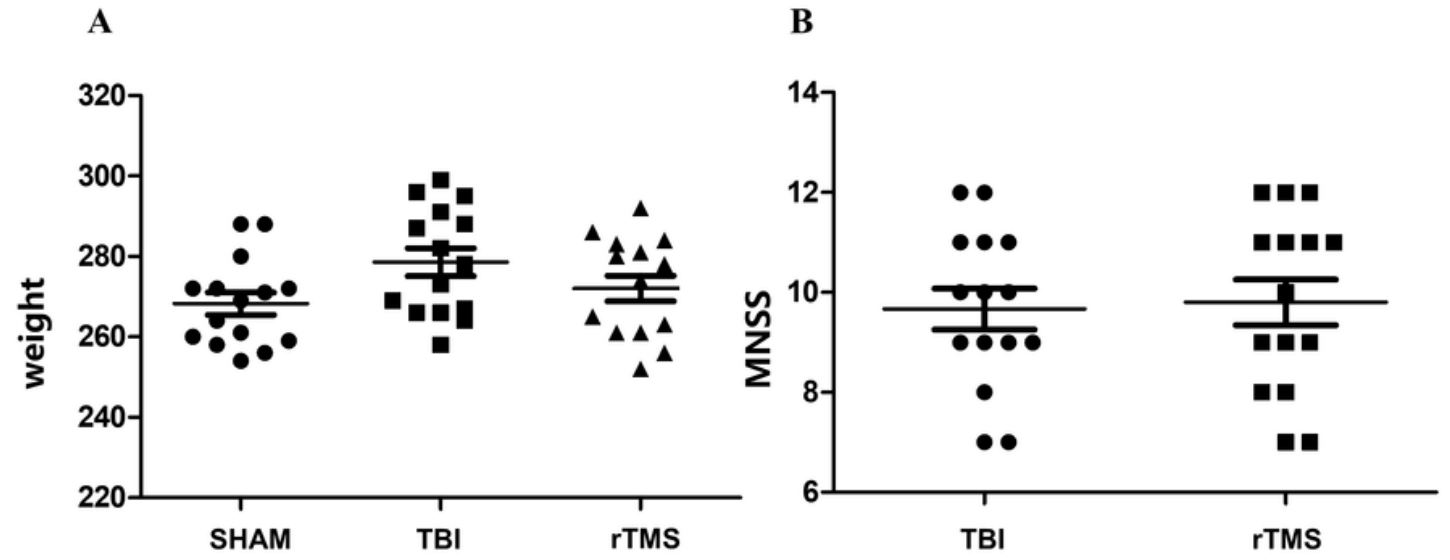

Figure 2

(A) body weight (B) MNSS values 


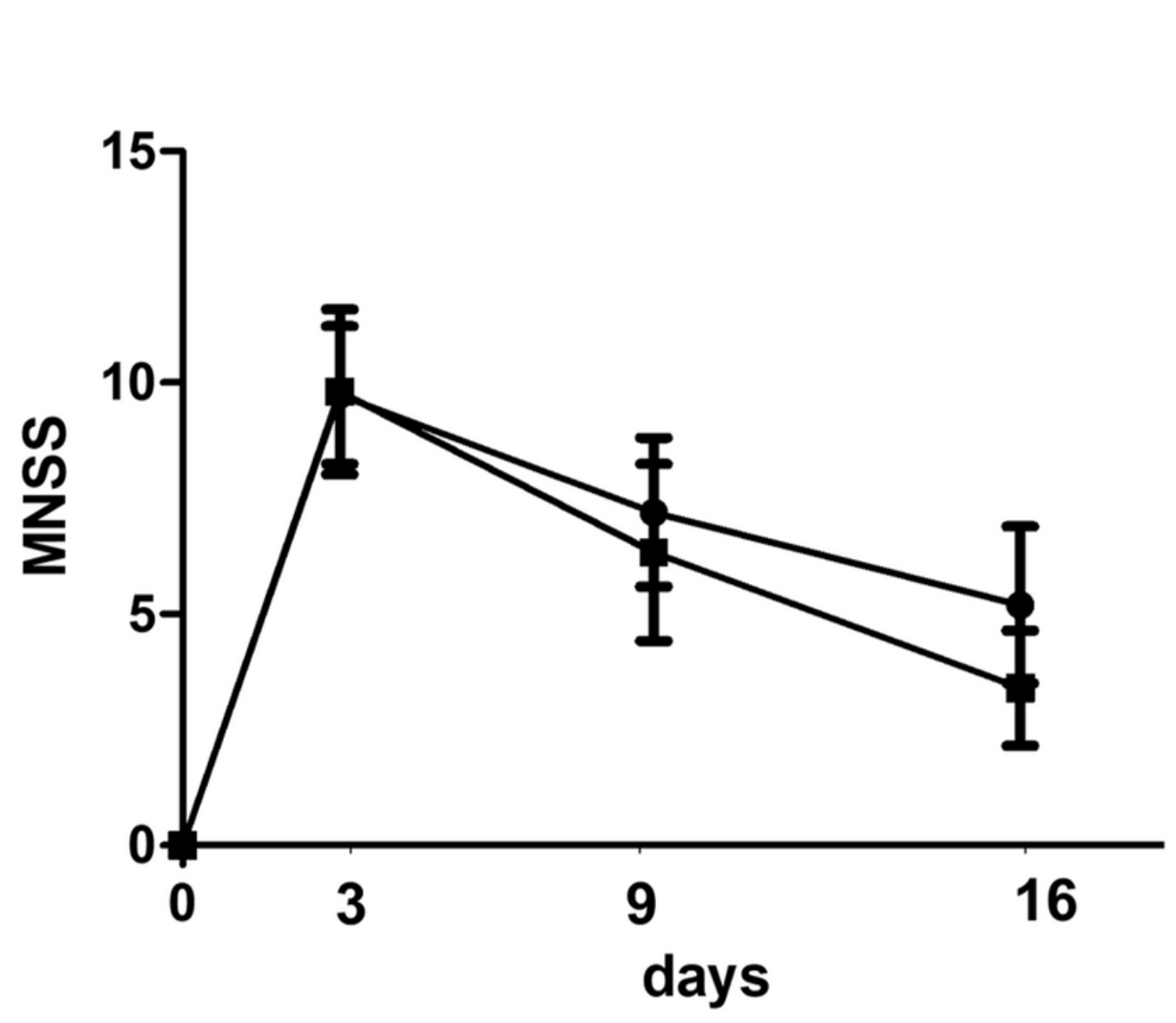

Figure 3

In the SHAM group, the MNSS value was 0 at any time. The MNSS values of the TBI group were 9.67 $\pm 1.59,7.20 \pm 1.61$, and $5.20 \pm 1.70$ at 3,9 , and 16 days after TBI, respectively. The MNSS values of the rTMS group were $9.80 \pm 1.78,6.33 \pm 1.92$, and $3.40 \pm 1.24$ at 3,9 , and 16 days after TBI, respectively. The MNSS value in the rTMS group was significantly lower than that in the TBI group at 16 days after TBI $(P<0.05)$. However, there was no significant difference in MNSS values at 3 or 9 days after TBI between the rTMS and TBI groups $(P>0.05)$. 


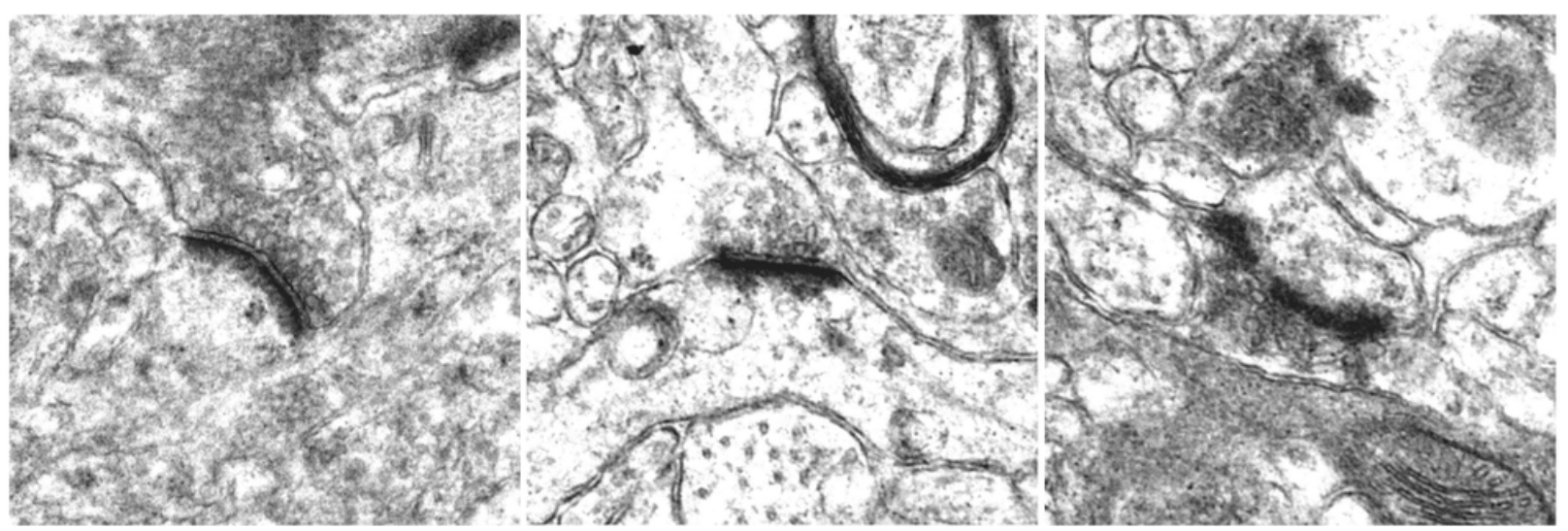

\section{Figure 4}

Ultra-structure of the cerebral cortex as shown by transmission electron microscopy $(20,000 x$ magnification). Data are shown in Table 1.

SHAM

A

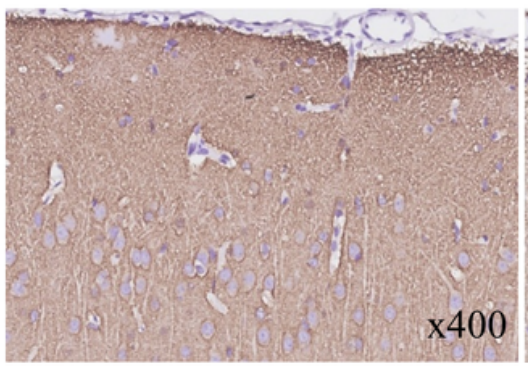

B

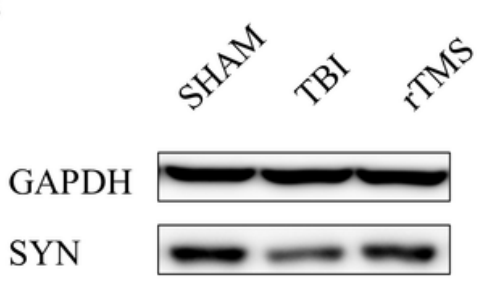

TBI

rTMS
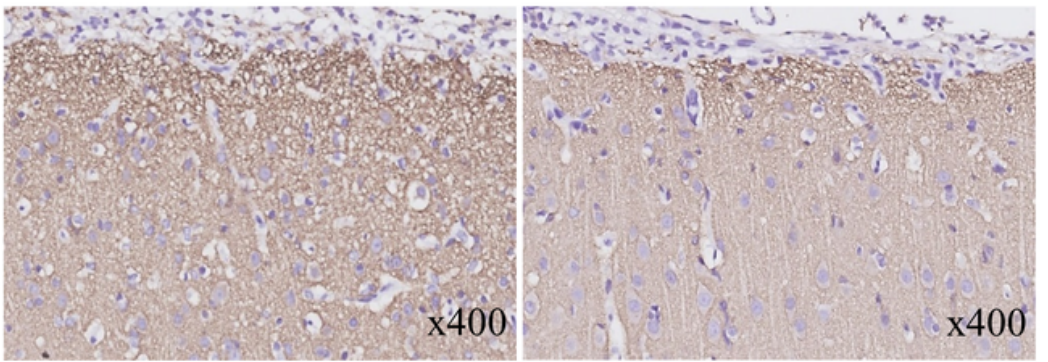

C

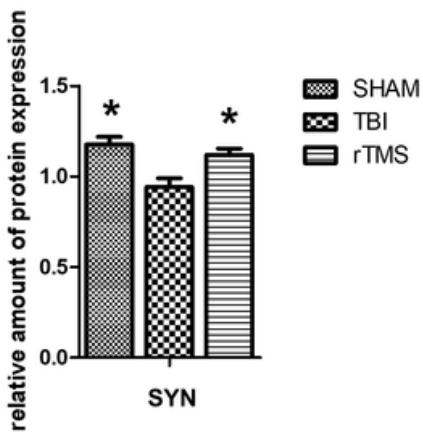




\section{Figure 5}

(A) After $20 \mathrm{~Hz}$ rTMS, there were more positive cells of SYN around the injury site compared with the TBI group. (B) Western blot images of the SYN protein level in the ipsilateral cortex at 16 days after TBI. Densitometry analysis of SYN bands corresponding to GAPDH. (C) Quantification of SYN mRNA expression in the ipsilateral hemisphere excluding the damaged area. Values are mean $\pm S D ;{ }^{*} P<0.05$ versus the TBI group.

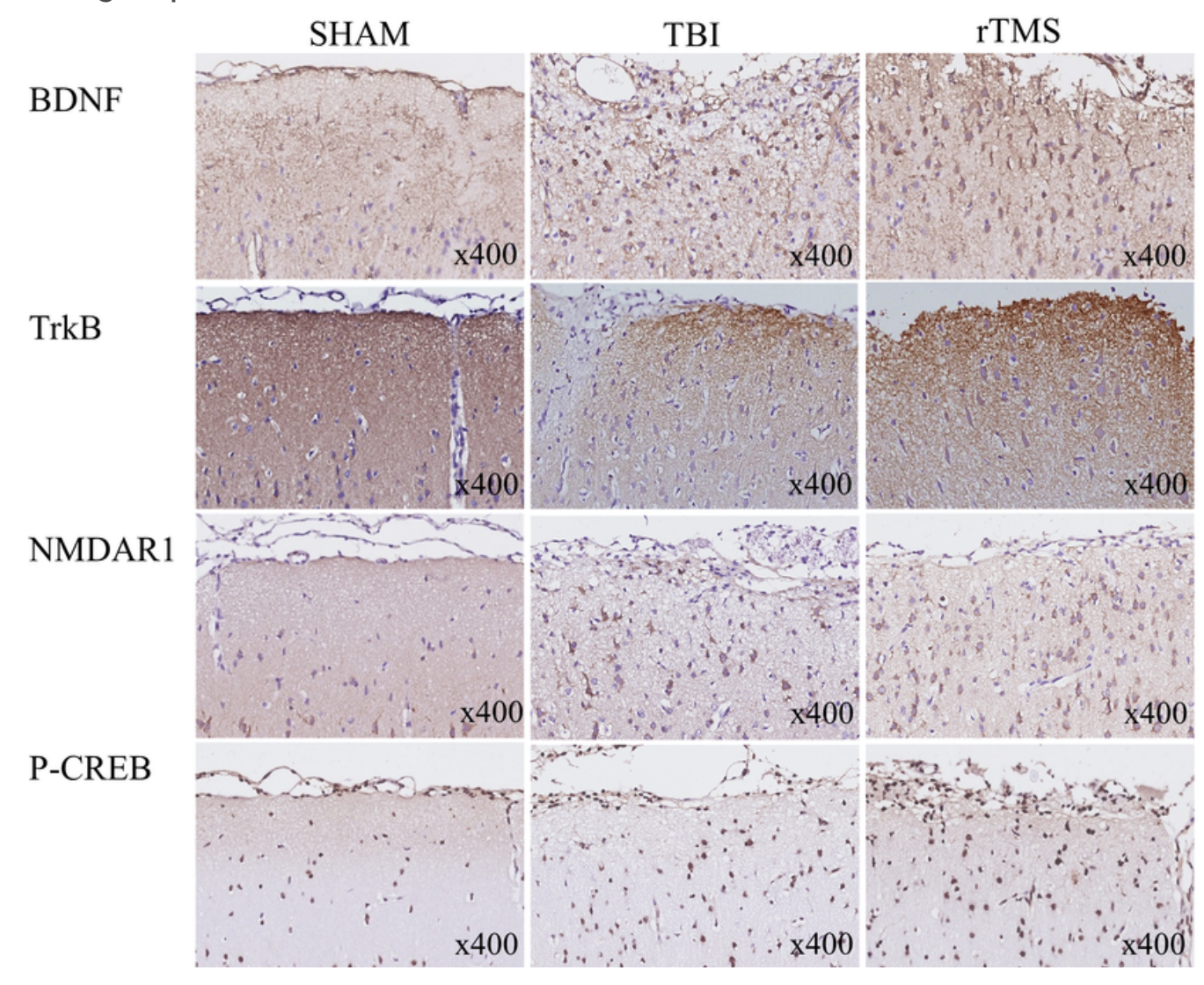

Figure 6

After $20 \mathrm{~Hz}$ rTMS, there were more positive cells of BDNF, TrkB, NMDAR1, and P-CREB around the injury site compared with the TBI group. 

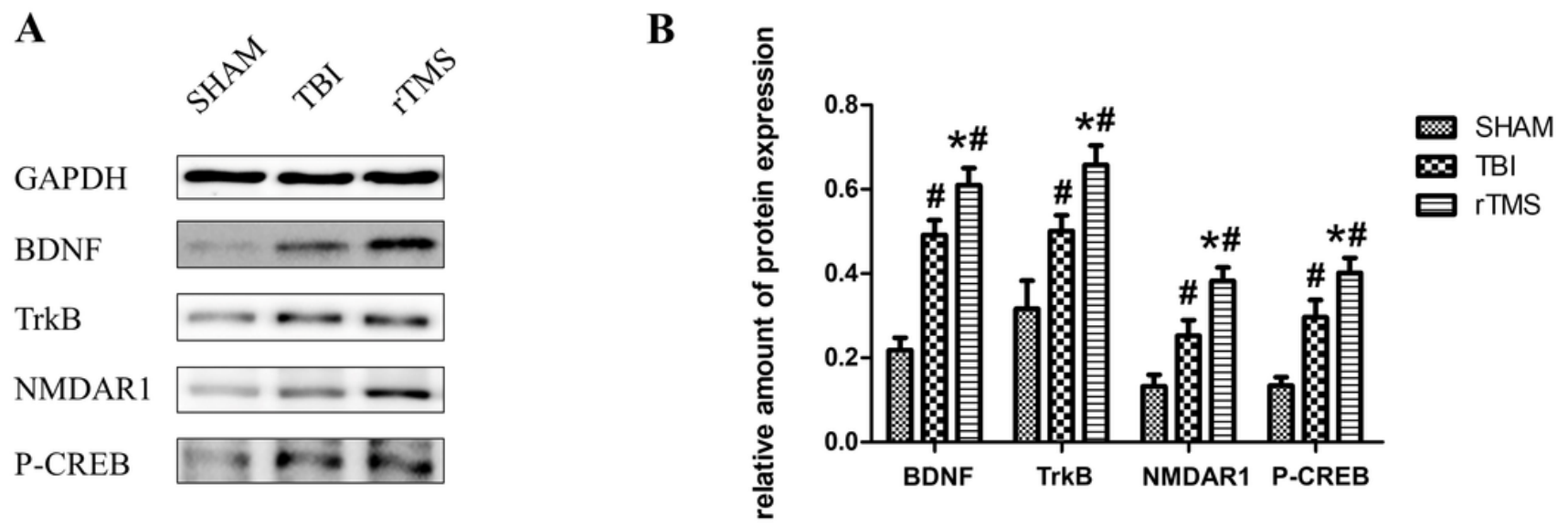

\section{Figure 7}

(A) Western blotting images representing protein expression levels of BDNF, TrkB, NMDAR1, and P-CREB in the ipsilateral cerebral cortex of rats at 16 days after TBI. (B) Densitometry analysis of BDNF, TrkB, NMDAR1, and P-CREB bands corresponding to GAPDH. Values are mean $\pm S D$; ${ }^{*}<0.05$ versus the $T B I$ group and $\# \mathrm{P}<0.05$ versus the sham group. 


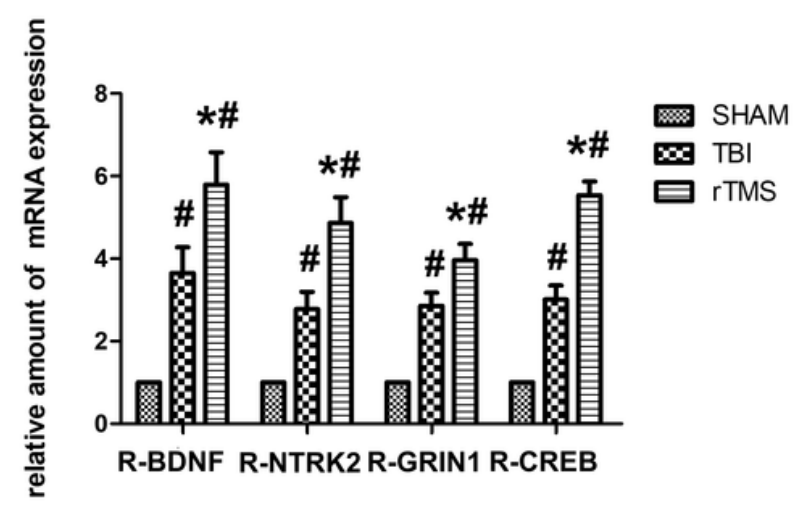

Figure 8

Quantification of BDNF, TrkB, NMDAR1, and P-CREB mRNA expression in the ipsilateral hemisphere excluding the damaged area. Values are mean $\pm S D$; ${ }^{*} P<0.05$ versus the TBI group and $\# P<0.05$ versus the sham group. 